\title{
Expression and clinical significance of Kelch-like epichlorohydrin-associated protein 1 in breast cancer
}

\author{
L. Zhang*, W.P. Yang*, L.Y. Wu, X. Zhu and C.Y. Wei \\ Department of Breast Surgery, \\ Affiliated Tumor Hospital of Guangxi Medical University, Nanning, China \\ *These authors contributed equally to this study. \\ Corresponding author: C.Y. Wei \\ E-mail: weichangyuan0723@163.com
}

Genet. Mol. Res. 15 (2): gmr.15027294

Received July 23, 2015

Accepted December 22, 2015

Published May 13, 2016

DOI http://dx.doi.org/10.4238/gmr.15027294

\begin{abstract}
Our objective was to explore the expression and clinical significance of Kelch-like epichlorohydrin-associated protein 1 (Keap1) in breast cancer tissue. Eighty-one breast cancer patients having undergone surgical treatment in our hospital between March 2002 and December 2008 were enrolled in this study. Normal tissue adjacent to tumors was used for the control samples. Diagnoses for all patients were confirmed by postoperative pathological examination. Immunohistochemical assays were used to measure the expression of Keap1 protein in breast cancer tissue and adjacent normal tissue, and its clinical significance was explored. We observed that $24.6 \%$ breast cancer tissue samples were positive for Keap1, a significantly lower proportion than that seen with adjacent normal tissue specimens $(80.2 \%$; $\mathrm{P}<0.05)$. The presence of Keap1 expression did not correlate with age, tumor size, pathological classification, or degree of differentiation. However, it was found to be significantly associated with tumor-nodemetastasis stage and the presence of lymphatic metastasis. KaplanMeier survival analysis showed a remarkably higher five-year survival
\end{abstract}


rate among patients with positive Keap1 expression than in those lacking detectable levels of the protein $(\mathrm{P}=0.032)$. Keap1 expression is significantly decreased in breast cancer tissue; therefore, the early detection of its expression might have great significance in determining prognosis for breast cancer patients.

Key words: Breast cancer; Keap1; Prognosis

\section{INTRODUCTION}

Breast carcinoma is one of the most common malignant tumors among women. With the ageing of the Chinese population, breast cancer incidence rises each year, and this disease has become a serious health threat to women (Zheng et al., 2013). As symptoms in the early stage of breast cancer are unremarkable, the majority of patients have already developed mid- or end-stage cancers by the time of diagnosis, having missed the optimal treatment time window, resulting in poor outcomes (Leal et al., 2015). Current treatments for breast cancer generally comprise comprehensive approaches, including surgery, radiotherapy, chemotherapy, and biological targeted and hormone therapy (Eisen et al., 2015). Although these treatments have improved breast cancer prognosis to a certain extent, the five-year survival rate remains relatively low. One important reason behind this is a lack of knowledge regarding the physiopathological mechanisms underlying the occurrence, progression, and invasion of breast carcinoma, and a resulting shortage of specific treatment agents (Theocharis et al., 2015). Therefore, in-depth research into these mechanisms is of great significance for the treatment of this disease. It has previously been reported that Kelch-like epichlorohydrin-associated protein 1 (Keap1) is an important tumor suppressor. Mutation of the Keapl gene or deficient protein expression promotes the occurrence, progression, and metastasis of various tumors (Leinonen et al., 2014). In this study, Keap1 protein expression in breast cancer tissue was detected using immunohistochemical (IHC) methods. The correlation between such expression and patient prognosis was analyzed, with the hope of providing new ideas and approaches for breast cancer prevention and treatment.

\section{MATERIAL AND METHODS}

\section{General information}

This study included 81 breast cancer patients having undergone surgical treatment and having had a diagnosis confirmed by postoperative pathological examination in our hospital between March 2002 and December 2008. The average age of these patients was $61 \pm 8.9$ years. Normal tissue adjacent to the tumor was also collected from all patients to be used as a control. All diagnoses were confirmed by postoperative pathological examinations. Complete clinical data was available for each patient, all of whom signed informed consent forms. These data included general and clinical characteristics, such as name, age, auxiliary examination results before and after surgery, tumor-node-metastasis (TNM) stage, and treatment record. Patients having received chemotherapy or radiotherapy before surgery were excluded. This study was approved by the ethics committee of our hospital. 


\section{IHC and hematoxylin-eosin (H\&E) staining}

Specimens were resected during surgery and submerged in pre-chilled neutral formaldehyde. Samples were kept at $4^{\circ} \mathrm{C}$ for $24 \mathrm{~h}$ before conventional paraffin embedding and sectioning procedures were performed. Paraffin sections $(6 \mathrm{~mm}$ thick) were deparaffinized with xylene and hydrated using a graded ethanol series. Antigen retrieval was performed with an antigen retrieval solution, and the sections were blocked with rabbit immune serum, before being incubated with an anti-Keap1 antibody (1:50 dilution; Santa Cruz Biotechnology, Dallas, TX, USA) in a $37^{\circ} \mathrm{C}$ incubator for $2 \mathrm{~h}$. Following thorough washing with a phosphate-buffered saline (PBS) solution, the sections were then incubated with a secondary antibody for $1 \mathrm{~h}$. After further PBS washes, horseradish peroxidase-conjugated streptavidin was added and allowed to react for $15 \mathrm{~min}$. This was followed by additional washes with PBS and diaminobenzidine chromogenesis. Finally, hematoxylin counterstaining was carried out and the sections were observed under a microscope. Negative controls were prepared by substituting the primary antibody with PBS. Meanwhile, conventional H\&E staining was conducted by carefully following standard procedures.

IHC staining results were assessed by two experienced pathologists using a blind method. For each section, cells were counted in 15 fields at high magnification (400X). The positive rate was defined by the number of Keap1-positive cells per 100 cells. Positive results in IHC assays (appearing as yellow-brown granules in the cytoplasm) were principally determined according to two factors, following a previously published study (Huang et al., 2013). Cells were rated based on staining intensity as follows: zero points were assigned to colorless cells, 1 point to cells stained light yellow, 2 points to those stained yellow, and 3 points to yellow-brown cells. Cells that scored 0-2 were considered negative, and those that scored 3-7 positive. Samples with 0-5, 6-25, 26-75, or higher than 76\% Keap1-positive cells were considered negative, and slightly, moderately, and strongly positive, respectively. Samples in any of the latter three categories were considered positive.

\section{Statistical analysis}

The SPSS 17.0 software (SPSS Inc., Chicago, IL, USA) was used to process statistical data. Enumeration data were analyzed by chi-square tests, and the Kaplan-Meier method was applied for survival analysis. P values lower than 0.05 were considered to indicate significant differences.

\section{RESULTS}

\section{Keap1 expression in breast cancer tissue and adjacent normal tissue}

All breast cancer patients included in this study had pathologically confirmed diagnoses. Pathological results from representative breast cancer patients are shown in Figure 1. IHC assays showed that Keap1 protein was mainly distributed in the cytoplasm, which in positive cells was stained yellow-brown or brown. The extent of Keapl expression was significantly higher in adjacent normal tissue than in breast cancer tissue (Figure 2). Statistical analysis demonstrated that the proportion of Keap1-positive adjacent normal tissue samples was $80.2 \%$, while that for breast cancer tissue was only $24.6 \%$. Moreover, this difference was statistically significant (Table 1). 

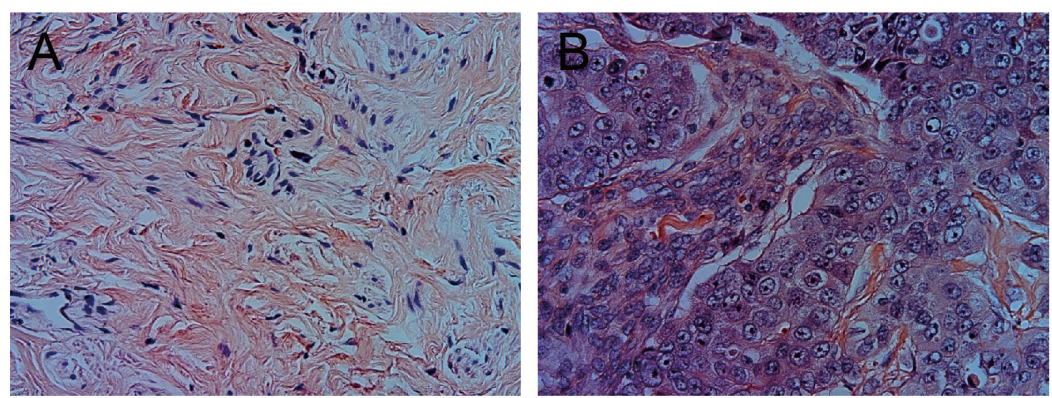

Figure 1. Hematoxylin-eosin staining of breast cancer tissue (lower image) and adjacent normal tissue (upper image).
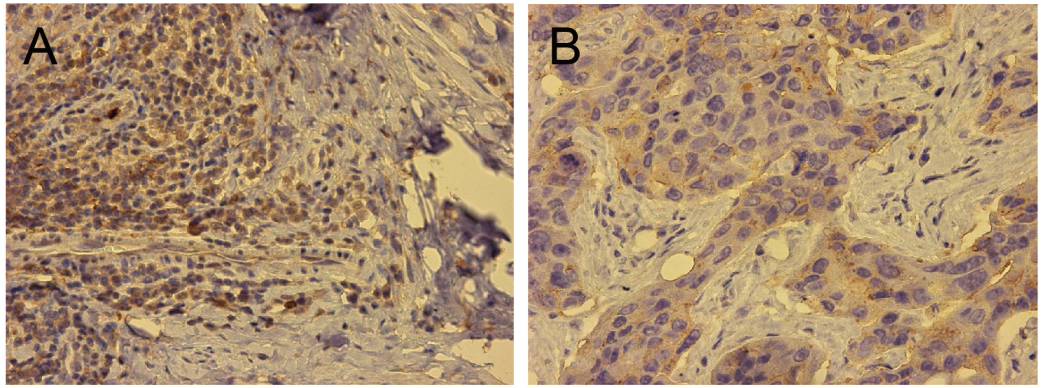

Figure 2. Representative images of Keap1 expression detected by immunohistochemical staining of breast cancer tissue (lower image) and adjacent normal tissue (upper image). Images were taken at 400X magnification.

Table 1. Summary of Keap1 expression in breast cancer tissue and adjacent normal tissue.

\begin{tabular}{l|c|c|c}
\hline \multirow{2}{*}{ Tissue type } & \multicolumn{2}{|c|}{ Keap1 expression } & \multirow{2}{*}{$\mathrm{P}$} \\
\cline { 2 - 3 } & Positive samples (\%) & Negative samples (\%) & \\
\hline Breast cancer & $20(24.6)$ & $61(75.4)$ & $<0.001$ \\
\hline Adjacent normal & $65(80.2)$ & $16(19.8)$ & \\
\hline
\end{tabular}

\section{Correlations between Keap1 expression and breast cancer clinicopathological classification}

Statistical analysis revealed that expression of Keap1 was not associated with age, tumor size, pathological classification, or degree of differentiation $(\mathrm{P}>0.05)$. However, it was remarkably correlated with TNM stage and the absence or presence of lymphatic metastasis $(\mathrm{P}<0.05$; Table 2).

\section{Correlation between Keap1 expression and clinical outcome}

Kaplan-Meier survival analysis indicated that patients demonstrating positive expression had notably higher five-year survival rates compared to those lacking Keap1 $(\mathrm{P}=$ 0.032; Figure 3). 
Table 2. Clinicopathological variables and Keap1 expression in breast carcinomas.

\begin{tabular}{|c|c|c|c|c|}
\hline \multirow[t]{2}{*}{ Group } & \multirow[t]{2}{*}{$\mathrm{N}$} & \multicolumn{2}{|c|}{ Keap1 } & \multirow[t]{2}{*}{$P$} \\
\hline & & Number of positive cases & Number of negative cases & \\
\hline \multicolumn{5}{|l|}{ Age (years) } \\
\hline$<50$ & 41 & 12 & 29 & \multirow[t]{2}{*}{0.33} \\
\hline$\geq 50$ & 40 & 8 & 32 & \\
\hline \multicolumn{5}{|l|}{ Tumor size } \\
\hline$<3 \mathrm{~cm}$ & 52 & 11 & 41 & \multirow[t]{2}{*}{0.83} \\
\hline$\geq 3 \mathrm{~cm}$ & 39 & 9 & 30 & \\
\hline \multicolumn{5}{|c|}{ Pathological classification } \\
\hline Ductal carcinoma & 39 & 8 & 31 & \multirow[t]{4}{*}{0.13} \\
\hline Lobular carcinoma & 30 & 8 & 22 & \\
\hline Medullary carcinoma & 8 & 2 & 6 & \\
\hline Mixed carcinoma & 4 & 3 & 1 & \\
\hline \multicolumn{5}{|l|}{ TNM stage } \\
\hline I & 21 & 11 & 10 & \multirow[t]{3}{*}{0.002} \\
\hline II & 31 & 6 & 25 & \\
\hline III & 29 & 3 & 26 & \\
\hline \multicolumn{5}{|c|}{ Degree of differentiation } \\
\hline High & 17 & 4 & 13 & \multirow[t]{3}{*}{0.68} \\
\hline Moderate & 34 & 7 & 27 & \\
\hline Low & 30 & 9 & 21 & \\
\hline \multicolumn{5}{|l|}{ Lymphatic metastasis } \\
\hline Yes & 34 & 13 & 22 & \multirow[t]{2}{*}{0.02} \\
\hline No & 47 & 7 & 39 & \\
\hline
\end{tabular}

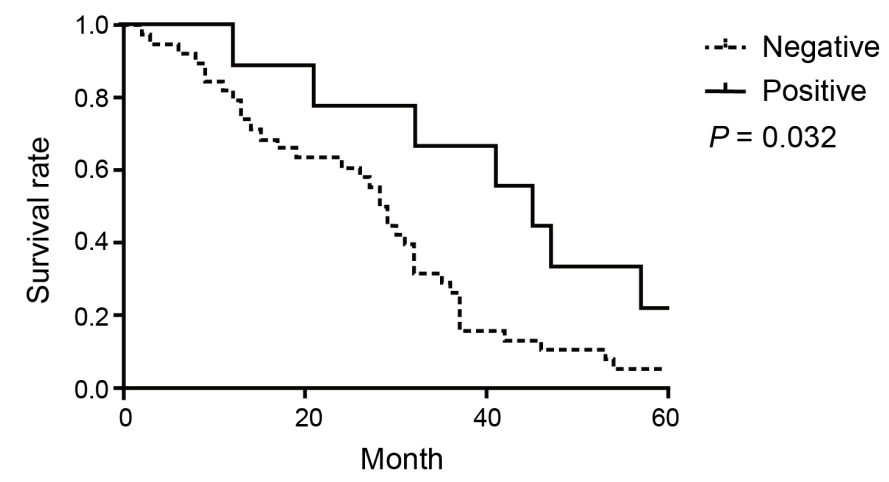

Figure 3. Keap1 expression was associated with overall survival.

\section{DISCUSSION}

Keap1 is a pivotal regulatory protein involved in the cellular anti-oxidation response (Chun et al., 2014). Under normal physiological conditions, Keap1 binds to erythroid-specific nuclear factor 2 (NF-E2)-related factor 2 (Nrf2) and anchors it to the actin cytoskeleton. This impedes the translocation of Nrf2 from the cytoplasm into the nucleus. In response to extracellular stimulation by electrophilic reagents or reactive oxygen species, Keap1 rapidly decouples from Nrf2. The liberated Nrf2 then moves into the nucleus, subsequently binding to Maf to generate a heterodimer. This heterodimer is able to recognize the antioxidant response element and promote gene transcription and expression of downstream phase II proteins, such as those of the proteasome (including ubiquitin), antioxidants, and detoxifying enzymes. 
Expression of these factors enhances the ability of cells to cope with oxidative stress. The Keap1/Nrf2 system plays an important role in tumor occurrence and development (Solis et al., 2010). Recent research has revealed that Keap1 is a key tumor suppressor involved in the occurrence and progression of various cancers. For example, non-small cell lung cancer patients expressing high levels of Keap1 in tumor tissue have been shown to benefit from significantly longer progression-free survival than those demonstrating low expression. The degree of Keap1 expression is also a critical factor affecting the efficacy of chemotherapy for lung cancer patients (Cao et al., 2012; Bauer et al., 2013). In addition, laryngeal and colon carcinoma patients with low Keap1 levels in tumor tissues show significantly lower five-year survival rates than those exhibiting elevated expression (Kansanen et al., 2013). The above studies suggest that the extent of Keapl expression plays an important role in the prognosis of various tumors. Expression of Nrf2 is increased in breast cancer tissue, and inhibiting the expression of this protein has been shown to suppress tumor development and invasion in in vitro cell culture experiments (Choi et al., 2014; Ryoo et al., 2015). Relevant studies on the expression of Keap1, which inhibits Nrf2, in breast cancer tissue are relatively scarce. In this study, the expression of Keap1 protein was detected in breast cancer tissue using IHC staining methods, and its relationship to clinicopathological characteristics and patient prognosis was explored.

This study revealed that Keap1, when detected, was primarily distributed in the cytoplasm. This protein was expressed in both breast cancer tissue and adjacent normal tissue, albeit at a significantly lower level in the former than in the latter. Further investigation revealed that the extent of its presence was closely correlated with TNM stage and the absence or presence of lymphatic metastasis. The level of Keap1 expression was unrelated to age, tumor size, and tumor pathological classification. These results suggest a central role for Keap1 in breast cancer metastasis. Kaplan-Meier survival analysis demonstrated that patients with increased Keap1 expression had significantly higher five-year survival rates than those in whom it was present at low levels, indicating that such expression exerts an important influence on breast cancer prognosis. In summary, these results suggest that the early detection of Keap 1 levels might be of great significance for cancer prognosis. In future research, the role of this protein in the occurrence and progression of breast cancer should be further validated by in vitro cell culture and in vivo animal experiments. Such investigations may provide new treatment targets for breast cancer and ultimately improve clinical outcomes. This study had some limitations, such as its relatively small sample size, sample selection bias, and singlecenter nature. Therefore, multicenter studies with larger sample sizes should be conducted to investigate this topic in the future.

Keap 1 expression is remarkably decreased in breast cancer tissue and closely correlated with TNM stage, lymphatic metastasis, and clinical prognosis, indicating its importance in determining breast cancer prognosis.

\section{Conflicts of interest}

The authors declare no conflict of interest.

\section{REFERENCES}

Bauer AK, Hill T, 3rd and Alexander CM (2013). The involvement of NRF2 in lung cancer. Oxid. Med. Cell. Longev. 2013: 746432. http://dx.doi.org/10.1155/2013/746432

Genetics and Molecular Research 15 (2): gmr.15027294 
Cao B, Zhu X, Chen S, Xiao Y, et al. (2012). [Keap1 expression for predicting the chemoresistance and prognosis of advanced non-small cell lung cancer]. Zhongguo Fei Ai Za Zhi 15: 591-596.

Choi BH, Ryoo IG, Kang HC and Kwak MK (2014). The sensitivity of cancer cells to pheophorbide a-based photodynamic therapy is enhanced by Nrf2 silencing. PLoS One 9: e107158. http://dx.doi.org/10.1371/journal.pone.0107158

Chun KS, Kundu J, Kundu JK and Surh YJ (2014). Targeting Nrf2-Keap1 signaling for chemoprevention of skin carcinogenesis with bioactive phytochemicals. Toxicol. Lett. 229: 73-84. http://dx.doi.org/10.1016/j. toxlet.2014.05.018

Eisen A, Fletcher GG, Gandhi S, Mates M, et al.; members of the Early Breast Cancer Systemic Therapy Consensus Panel (2015). Optimal systemic therapy for early breast cancer in women: a clinical practice guideline. Curr. Oncol. 22 (Suppl 1): S67-S81.http://dx.doi.org/10.3747/co.22.2320

Huang CF, Zhang L, Ma SR, Zhao ZL, et al. (2013). Clinical significance of Keap1 and Nrf2 in oral squamous cell carcinoma. PLoS One 8: e83479. http://dx.doi.org/10.1371/journal.pone.0083479

Kansanen E, Kuosmanen SM, Leinonen H and Levonen AL (2013). The Keap1-Nrf2 pathway: Mechanisms of activation and dysregulation in cancer. Redox Biol. 1: 45-49. http://dx.doi.org/10.1016/j.redox.2012.10.001

Leal F, Liutti VT, Antunes dos Santos VC, Novis de Figueiredo MA, et al. (2015). Neoadjuvant endocrine therapy for resectable breast cancer: A systematic review and meta-analysis. Breast 24: 406-412. http://dx.doi.org/10.1016/j. breast.2015.03.004

Leinonen HM, Kansanen E, Pölönen P, Heinäniemi M, et al. (2014). Role of the Keap1-Nrf2 pathway in cancer. $A d v$. Cancer Res. 122: 281-320. http://dx.doi.org/10.1016/B978-0-12-420117-0.00008-6

Ryoo IG, Choi BH and Kwak MK (2015). Activation of NRF2 by p62 and proteasome reduction in sphere-forming breast carcinoma cells. Oncotarget 6: 8167-8184. http://dx.doi.org/10.18632/oncotarget.3047

Solis LM, Behrens C, Dong W, Suraokar M, et al. (2010). Nrf2 and Keap1 abnormalities in non-small cell lung carcinoma and association with clinicopathologic features. Clin. Cancer Res. 16: 3743-3753. http://dx.doi.org/10.1158/10780432.CCR-09-3352

Theocharis AD, Skandalis SS, Neill T, Multhaupt HA, et al. (2015). Insights into the key roles of proteoglycans in breast cancer biology and translational medicine. Biochim. Biophys. Acta 1855: 276-300.

Zheng Y, Wu CX and Zhang ML (2013). The epidemic and characteristics of female breast cancer in China. China Oncol 23: 561-569. 